\title{
Functional Characterisation and Antimicrobial Efficiency Assessment of Smart Nanohydrogels Containing Natamycin Incorporated into Polysaccharide-Based Films
}

\author{
Clara Fuciños ${ }^{1,2}$ - Martín Míguez ${ }^{1}$. Miguel A. Cerqueira ${ }^{2}$. \\ Maria J. Costa ${ }^{2}$ - António A. Vicente ${ }^{2}$ - María L. Rúa ${ }^{1}$. \\ Lorenzo M. Pastrana ${ }^{1}$
}

Received: 3 November 2014 / Accepted: 11 March 2015 /Published online: 24 March 2015

(C) Springer Science+Business Media New York 2015

\begin{abstract}
The potential application of polysaccharide-based films containing smart nanohydrogels for the controlled release of food preservatives is demonstrated here. Smart active packaging is the most promising alternative to traditional packaging as it provides a controlled antimicrobial effect, which allows reducing the amount of preservatives in the food bulk, releasing them only on demand. This work evaluates the usefulness of smart thermosensitive poly $(N$ isopropylacrylamide) (PNIPA) nanohydrogels with or without acrylic acid (AA) incorporated into polysaccharide-based films (GA) to transport natamycin and release it as a response to environmental triggers. Release kinetics in liquid medium from GA films containing PNIPA/AA nanohydrogels (GAPNIPA(5) and GA-PNIPA-20AA(5)) presented a characteristic feature regarding the films without nanohydrogels that was the appearance of a lag time in natamycin release, able to reach values of around $35 \mathrm{~h}$. Another important feature of natamycin release kinetics was the fact that the release from GA-PNIPA/AA films only occurred when temperature was increased, so that the natamycin release was restricted to when there is a risk of growth of microorganisms that cause food spoilage or the development of pathogenic microorganisms. Additionally, it could be observed that the relative fraction of natamycin released from GA-PNIPA/AA films was significantly $(p<0.05)$ higher than that released from GA films
\end{abstract}

Clara Fuciños

clarafg@gmail.com

1 Biotechnology Group, Department of Analytical Chemistry and Food Science, University of Vigo, As Lagoas s/n, 32004 Ourense, Spain

2 Centre of Biological Engineering, University of Minho, Campus de Gualtar, 4710-057 Braga, Portugal loaded with the same amount of free natamycin. It can be hypothesised that the encapsulation of natamycin into nanohydrogels helped it to be released from GA films, creating reservoirs of natamycin into the films and, therefore, facilitating its diffusion through the film matrix when the nanohydrogel collapses. In a solid medium, the low water availability limited natamycin release from GA-PNIPA/AA films restricting the on/off release mechanism of PNIPA/AA nanohydrogels and favouring the hydrophobic interactions between natamycin and polymer chains at high temperatures. Despite the low natamycin release in solid media, antimicrobial efficiency of GA-PNIPA(5) films containing natamycin in acidified agar plates was higher than that obtained with GA films without natamycin and GA films with free natamycin, probably due to the protecting effect against degradation when natamycin was included in the nanohydrogels, allowing its release only when the temperature increased.

Keywords K-Carrageenan · Locust bean gum · Edible film · Poly ( $N$-isopropylacrylamide) nanohydrogels .

Controlled release $\cdot$ Natamycin $\cdot$ Active packaging

\begin{tabular}{|c|c|}
\hline \multicolumn{2}{|l|}{ Nomenclature } \\
\hline AA & Acrylic acid \\
\hline AP & Aqueous phase \\
\hline Atlas ${ }^{\mathrm{TM}} \mathrm{G}-1086$ & $\begin{array}{l}\text { PEG-40 sorbitol } \\
\text { hexaoleate }\end{array}$ \\
\hline DL & Detection limit \\
\hline$F_{t}$ & $\begin{array}{l}\text { Fraction of natamycin } \\
\text { released from films } \\
\text { towards agar plates }\end{array}$ \\
\hline GA-NA & GA films containing free natamycin \\
\hline
\end{tabular}




GA-PNIPA-
20AA(5)
GA-PNIPA-
20AA(5)+NA(8)
GA-PNIPA(5)
GA-
PNIPA(5)+NA( $)$ GA-PNIPA/AA
GA
GA
GA+NA(564)
GA+NA(8)
Isopar ${ }^{\mathrm{TM}} \mathrm{M}$
LCST
MIC
$n$

NIPA
NMBA
OP
P 1.01
PDA
PNIPA-20AA(5)

PNIPA

PNIPA(5)

PNIPA/AA

RP-HPLC

Sc 1.02

$\operatorname{Span}^{\mathrm{TM}} 83$
Polysaccharide-based

films containing PNIPA-20AA(5)

nanohydrogel

GA films containing

PNIPA-20AA(5) nanohydrogels

loaded with $7.92 \mu \mathrm{g}$ of natamycin

Polysaccharide-based

films containing PNIPA(5)

nanohydrogel

GA films containing

PNIPA(5) nanohydrogels loaded

with $7.92 \mu \mathrm{g}$ of natamycin

Polysaccharide-based

films containing poly

( $N$-isopropylacrylamide)

nanohydrogel with or

without copolymerised acrylic acid

Polysaccharide-based films

GA films containing $564.24 \mu \mathrm{g}$

of free natamycin

GA films containing

$7.92 \mu \mathrm{g}$ of free natamycin

Isoparaffinic synthetic hydrocarbon

Lower critical solution temperature

Minimum inhibitory

concentrations

The diffusional exponent

that depends on both the

geometry of the delivery

system and the physical

mechanisms involved

$\mathrm{N}$-isopropylacrylamide

$N, N^{\prime}$-methylenebisacrylamide

Oil phase

Penicillium commune

Potato dextrose agar

Poly( $N$-isopropylacrylamide)

nanohydrogel copolymerised

with $20 \%(w / w)$ of acrylic

acid using $5 \%(w / w)$ of

crosslinking

Poly( $N$-isopropylacrylamide)

Poly( $N$-isopropylacrylamide)

nanohydrogel with $5 \%$

$(w / w)$ of crosslinking

Poly( $N$-isopropylacrylamide)

nanohydrogel with or without

copolymerised acrylic acid

Reverse phase

high-performance liquid

chromatography

Saccharomyces cerevisiae

Sorbitan sesquiolate
ST
$v_{\max }$
$\gamma F_{\max }$
Surfactant
Maximum release rate $\left(\mathrm{h}^{-1}\right)$
Maximum relative
fraction of natamycin
released from films
$\gamma F_{t}$
$\lambda$
Relative fraction of
natamycin released from films
The lag time for the natamycin
release, expressed in hours (h)

\section{Introduction}

In recent years, considerable importance has been given to the development of new materials for food packaging applications. One of the main objectives in the food industry is to replace the traditional food packaging, designed to protect food against the effects of the environment (e.g. microorganisms, oxygen, off-odours and light), where the key safety objective is for it to be as inert as possible (i.e. there should be a minimum of interaction between the food and the packaging) (Dainelli et al. 2008) by multifunctional packaging (e.g. active and smart packaging) (Han 2005; Han et al. 2005; Rooney 2005). The most innovative developments in the area of food packaging propose a multifunctional packaging based on the interaction between the package materials, the food and the environment, leading to a dynamic role in food preservation and quality and consumers safety (Brody et al. 2008; Dainelli et al. 2008). This group of multifunctional packaging can comprise two types of packaging: (a) active packaging with the purpose of extending the shelf life of food whilst maintaining (or even improving) its quality (Vermeiren et al. 1999) and (b) smart packaging which responds to environmental conditions (e.g. temperature and $\mathrm{pH}$ ) increasing food protection (e.g. by releasing an antimicrobial) or alerting the consumers to contamination and/or the presence of pathogens (Sekhon 2010).

Natamycin (pimaricin) is a polyene antifungal compound produced by Streptomyces natalensis and related species. It was approved as Generally Recognised As Safe (GRAS) by the Food and Drug Administration (FDA) in the USA and as a natural preservative in the European Union (E235). It is largely used in the food industry as a preservative on the surface of cheese, fruit and other non-sterile products, such as meat and sausages (Farid et al. 2000; Vanden Bossche et al. 2003). However, natamycin presents some technological problems associated with its use as food preservative: low solubility in water and low chemical stability in acidic conditions and under light radiation (Koontz et al. 2003; Stark and Tan 2003).

In previous works (Fuciños et al. 2012; Fuciños et al. 2014b), the limitations of active packaging systems for food applications have been addressed. Most of these active packaging systems include passive diffusion systems (Türe et al. 
2009; Fajardo et al. 2010; Hanušová et al. 2010; Bierhalz et al. 2012) in which an antimicrobial compound release is controlled by the concentration difference between the food, the surrounding medium and packaging materials, thus resulting in an uncontrolled release of antimicrobial compounds. In Fuciños et al. (2012, 2014b), the use of smart poly( $N$ isopropylacrylamide) (PNIPA) nanohydrogels has been proposed as an alternative to passive diffusion mechanism, which allows a controlled release of bioactive molecules (i.e. natamycin) as a response to environmental changes (temperature and/or $\mathrm{pH}$ ). Thus, the amount of preservative in the food could be reduced. These smart PNIPA nanohydrogels have a hydrophilic/hydrophobic balance in the side chains that allows the formation of hydrogen bonds between water molecules and hydrophilic groups of the hydrogel. Below its lower critical solution temperature (LCST), nanohydrogels remain soluble and swelled and, in this state, can be loaded with preservative. The value of LCST for PNIPA polymers is around $33{ }^{\circ} \mathrm{C}$ (Schild 1992; Zhang et al. 2002; Schmaljohann 2006). When the temperature increases above the LCST, the hydrophobic groups are strengthened dramatically leading to the collapse of the polymer chains (Schild 1992; Zhang et al. 2002; Cordeiro et al. 2009; Fuciños et al. 2014a), and the release of the preservative occurs. By varying the composition of PNIPA nanohydrogels, it is possible to easily modulate the polymer collapse pattern. Fuciños et al. (2014a) showed how the copolymerisation of PNIPA with acrylic acid (AA) imparts different structural properties to nanohydrogels affecting the particle size and swelling capacity. In the same manner, natamycin release kinetics were very different, depending on the nanohydrogel composition (Fuciños et al. 2014b). PNIPA nanohydrogel copolymerised with AA allowed a slower but more continuous release of natamycin. Thus, the antimicrobial efficiency of natamycin was improved when encapsulated in PNIPA/AA nanohydrogels due to the protection that the nanohydrogel gives against environmental degradation whilst also lowering the presence of preservatives in food bulk (Fuciños et al. 2012). But to apply them to the food surface, a carrier or support of loaded nanoparticles such as an edible film or a coating may be helpful.

Considering food packaging as one of the main potential applications of these smart nanohydrogels, in Cerqueira et al. (2014), natamycin-loaded nanohydrogels were successfully incorporated into polysaccharide-based films without influencing their main physical properties. So it seems that this packaging system could allow improving by, on the one hand, applying active agents onto the food surface by film formation and, on the other hand, by achieving a controlled release of the preservative only when it is necessary, due to nanohydrogel behaviour under temperature changes.

In this work, natamycin release kinetics from nanohydrogels incorporated into polysaccharide-based films (GA-PNIPA/AA) were evaluated, under different temperatures and media, in free state or when encapsulated in nanohydrogels. Additionally, the antimicrobial efficiency of these new active and smart films was assessed in a food model system.

\section{Materials and Methods}

\section{Preparation of Natamycin-Loaded PNIPA/AA Nanohydrogels}

The synthesis of PNIPA/AA nanohydrogels and the preparation of natamycin-loaded PNIPA/AA nanohydrogels were performed as presented by Fuciños et al. (2012). For the synthesis of nanohydrogels, a water in oil (W/O) microemulsion formed by $58 \%(w / w)$ of aqueous phase (AP-80\%,w/w, water and $20 \%, w / w$, monomer), $17 \%(w / w)$ of oil phase (OP-isoparaffinic synthetic hydrocarbon: Isopar ${ }^{\mathrm{TM}}, 98 \%$, $v / v$, Quimidroga, S.A., Barcelona, Spain) and $25 \%(w / w)$ of surfactant (ST-PEG-40 sorbitol hexaoleate: Atlas ${ }^{\mathrm{TM}} \mathrm{G}-1086$ and sorbitan sesquiolate: $\operatorname{Span}^{\mathrm{TM}} 83$, Croda Ibérica, S.A., Barcelona, Spain) was prepared. For pure PNIPA nanohydrogels (PNIPA(5)), the ratio $(w / w)$ of $N$ isopropylacrylamide (NIPA, $99 \%, w / w$, Acros Organics, Geel, Belgium) based on the monomers $\left(\mathrm{m}_{\mathrm{NIPA}} / \mathrm{m}_{\text {monomer }}\right)$ was 1. For NIPA copolymerised with AA (stabilised with hydroquinone monomethyl ether, for synthesis, Merck, Darmstadt, Germany) (PNIPA-20AA(5)) nanohydrogels, $\mathrm{m}_{\mathrm{NIPA}} / \mathrm{m}_{\text {monomer }}$ was 0.80 and $\mathrm{m}_{\mathrm{AA}} / \mathrm{m}_{\text {monomer }}$ was 0.20 . To preserve the shape and size of the particles during handling, we used the $N, N^{\prime}$-methylenebisacrylamide (NMBA) crosslinking agent for synthesis (Merck, Darmstadt, Germany) at a ratio $\mathrm{m}_{\mathrm{NMBA}} / \mathrm{m}_{\text {monomer }}=0.05$.

The phases (AP and OP) were solubilised and mixed in a $100-\mathrm{mL}$ reactor equipped with mechanical stirring and thermostatised at $25^{\circ} \mathrm{C}$. The reaction medium was purged by bubbling nitrogen to eliminate oxygen. Polymerisation was triggered by adding the initiator $\left(\mathrm{NaHSO}_{3}\right.$ for analysis, Merck, Darmstadt, Germany) at a ratio $\mathrm{m}_{\mathrm{NaHSO} 3} / \mathrm{m}_{\text {monomer }}=$ 0.01 and monitored by the temperature increase inside the glass reactor. Chloroform (Panreac, Barcelona, Spain) was used to selectively precipitate inverse micelles formed during microemulsion, which contain synthesised nanoparticles. This mixture was poured over diethyl ether (Panreac, Barcelona, Spain) to dissolve and remove any residual impurities. The pure polymer was separated from diethyl ether by decantation and then it was dried overnight in an oven $\left(50^{\circ} \mathrm{C}\right)$ and then ground in a colloid mill (IKA-Werke GmbH \& Co. KG, Staufen, Germany).

Natamycin-loaded PNIPA/AA nanohydrogels were prepared by dispersing, with agitation, the PNIPA/AA nanohydrogel powder in distilled water for $3 \mathrm{~h}$ at room temperature to allow the nanoparticles to swell properly. This 
suspension was then mixed with a natamycin $(50 \%(w / w)$ lactose, VGP Pharmachem, Barcelona, Spain) water solution to obtain final concentrations of $12.5 \mathrm{mg} \mathrm{mL}^{-1}$ of nanohydrogel and $0.4 \mathrm{mg}$ of commercial natamycin powder per $\mathrm{mL}\left(21.93 \mu \mathrm{g} \mathrm{mL}^{-1}\right.$ of solubilised pure natamycin). The mixture was then stirred for $12 \mathrm{~h}$ at $25^{\circ} \mathrm{C}$ to guarantee the incorporation of natamycin into the nanohydrogel particles (Fuciños et al. 2014b).

\section{Preparation of Natamycin-Loaded PNIPA/AA Nanohydrogels Incorporated into $\kappa$-Carrageenan and Locust Bean Gum (GA) Films}

Polysaccharide film-forming solutions were prepared as described in Cerqueira et al. (2014). Briefly, 0.4 and $0.6 \%(w / v)$ of K-carrageenan (Gelcarin DX5253, FMC Biopolymer, Norway) and locust bean gum (Genu gum type RL-200, CP Kelco, USA), respectively, were suspended in distilled water under agitation during $1 \mathrm{~h}$ at $25^{\circ} \mathrm{C}$, after which $0.3 \%(w / v)$ of glycerol ( $87 \%, v / v$, Panreac, Spain) was added to the solution. Then, the film-forming solutions were homogenised at $80^{\circ} \mathrm{C}$ under agitation during $30 \mathrm{~min}$. Natamycin and the natamycinloaded PNIPA/AA nanohydrogels were added after the decrease of the temperature to $30{ }^{\circ} \mathrm{C}$ in order to avoid the nanohydrogel collapse.

Two different concentrations of free natamycin were evaluated: $500 \mu \mathrm{g} \mathrm{mL}^{-1}$, based on the published work by Fajardo et al. (2010), and $21.93 \mu \mathrm{g} \mathrm{mL}^{-1}$, based on the concentration of solubilised pure natamycin charged into the nanohydrogels. Based on these concentrations and assuming that natamycin was distributed uniformly in the film, the final amount of natamycin in the films (with $35 \mathrm{~mm}$ of diameter) was estimated to be 564.24 and $7.92 \mu \mathrm{g}$, respectively (films prepared with free natamycin). These films are mentioned as GA+NA(564) and GA+NA(8), respectively. Natamycin-loaded nanohydrogels, prepared as explained in the section "Preparation of Natamycin-Loaded PNIPA/AA Nanohydrogels", were added to films to a final concentration of $0.2 \%(v / v)$. The final amount of natamycin in each film with $35 \mathrm{~mm}$ of diameter was estimated to be $7.92 \mu \mathrm{g}$. These films are mentioned as GA-PNIPA(5)+NA(8) and GAPNIPA-20AA(5)+NA(8) for the two different nanohydrogels used in this work.

The compounds were added and stirred until a homogeneous solution was obtained. Then, $28 \mathrm{~mL}$ of solution was cast into polystyrene Petri dishes and dried at $25{ }^{\circ} \mathrm{C}$ during $48 \mathrm{~h}$, resulting in films with $0.045 \pm 0.002 \mathrm{~mm}$ of thickness. In the same manner, films without the incorporation of any compound were prepared and used as control and mentioned as GA films. All films were conditioned in desiccators containing a saturated solution of $\mathrm{Mg}\left(\mathrm{NO}_{3}\right)_{2} \cdot 6 \mathrm{H}_{2} \mathrm{O}$ at $53 \%$ relative humidity (RH) and $20{ }^{\circ} \mathrm{C}$.

\section{Natamycin Release from PNIPA/AA Nanohydrogels Incorporated into GA Films}

\section{Liquid Medium}

The procedure for studying the natamycin release from nanohydrogels in a liquid medium was based on that described in Fuciños et al. (2012). GA films with $35 \mathrm{~mm}$ diameter submerged in $3 \mathrm{~mL}$ of distilled water were placed in a dialysis membrane (MWCO $3500 \mathrm{~g} \mathrm{~mol}^{-1}$, SnakeSkin ${ }^{\mathrm{TM}}$, Pierce, Rockford, IL, USA) and dialyzed against $12 \mathrm{~mL}$ of distilled water at 10 and $37^{\circ} \mathrm{C}$, with constant stirring. Samples of $1 \mathrm{~mL}$ were taken at intervals from the solution in order to determine the natamycin concentration. The volume removed from the probe was returned after measurements had been made in order to maintain a constant volume. Two replicates of each film were evaluated for each temperature.

In order to evaluate natamycin availability in the liquid medium, a control experiment was performed, quantifying natamycin amount in distilled water, initially added with an aqueous solutions of commercial natamycin (with the same amounts of natamycin employed to be incorporated into the films), and incubated at 10 and $37{ }^{\circ} \mathrm{C}$ (below and above, respectively, the LCST of nanohydrogel to allow its swollen and collapse, respectively). Aliquots were removed at different time intervals and natamycin amount was determined in the same way as natamycin released from films in the liquid medium (see "In Distilled Water" section). Three replicates were evaluated for each temperature.

\section{Solid Medium}

GA films with $35 \mathrm{~mm}$ diameter were placed on Petri dishes having the same diameter, which contained $2 \mathrm{~mL}$ of agar (bacteriological European type, Cultimed, Panreac, Barcelona, Spain) (12 mg mL $\mathrm{m}^{-1}$ ). Then, Petri dishes were incubated at two different temperatures, 5 and $37^{\circ} \mathrm{C}$, and $55 \% \mathrm{RH}$. At each sampling time, films were removed from the agar surface using a clamp. Agar samples were then sliced, frozen and then lyophilised to extract and quantify the natamycin released from GA films as explained in the section "In Agar Plates". Four replicates of each film were evaluated for each temperature.

In the same way as in the liquid medium, a control experiment was performed quantifying natamycin availability in agar plates initially added with an aqueous solution of commercial natamycin to obtain final concentrations in agar from 0.1 to $2.5 \mu \mathrm{g} \mathrm{mL}-1$, and incubated at 5 and $37{ }^{\circ} \mathrm{C}$ and $55 \%$ RH. Natamycin was extracted and quantified at different time intervals in the same way as natamycin released from films in the solid medium (see "In Agar Plates" section). Three replicates were evaluated for each temperature. 


\section{Natamycin Quantification}

\section{In Distilled Water}

The natamycin concentration in the dialysate (distilled water) was determined spectrophotometrically at $319 \mathrm{~nm}$ (Beckman Coulter Inc., Brea, CA, USA) as described in Fuciños et al. (2012) by the molar absorption coefficient $(\varepsilon)$ calculated for natamycin $\left(0.077 \pm 0.006 \mathrm{~mL} \mathrm{\mu g}^{-1} \mathrm{~cm}^{-1}\right)$.

\section{In Agar Plates}

Samples with $2 \mathrm{~mL}$ of agar media containing the natamycin released from GA films were sliced, frozen and then lyophilised. After lyophilisation, samples were embedded in $2 \mathrm{~mL}$ of methanol (CHROMASOLV ${ }^{\circledR}$ for HPLC, $\geq 99.9 \%$, $v / v$, Sigma-Aldrich, Munich, Germany) during $1 \mathrm{~h}$ under agitation at $25^{\circ} \mathrm{C}$ in order to extract the natamycin without any additional dilution. Then, samples were filtered $(0.45 \mu \mathrm{m})$ in order to remove any remaining agar. In these extracts, natamycin was quantified by reverse phase highperformance liquid chromatography (RP-HPLC), following the method of Roberts et al. (2011), with some modifications. RP-HPLC was performed using an Agilent 1200 system (Agilent Technologies, Palo Alto, CA, USA). Separation was performed employing an $\mathrm{ACE}^{\circledR} \mathrm{C} 18$ column (4.6 mm $\times$ $150 \mathrm{~mm}, 5 \mu \mathrm{m}$ ) with the column oven kept at $30{ }^{\circ} \mathrm{C}$. The flow rate was $1.0 \mathrm{~mL} \mathrm{~min}^{-1}$, the injection volume was $20 \mu \mathrm{L}$ and the detection wavelength was $319 \mathrm{~nm}$. Mobile phase A consisted in Milli-Q water/acetic acid (puriss., p.a. ACS reagent, $\geq 99.8 \%, v / v$, Sigma-Aldrich, Munich, Germany) $(97: 3, v / v)$ and mobile phase B consisted of methanol/acetic acid $(97: 3, v / v)$. Mobile phase A was maintained at $90 \%(v / v)$ for the first $6 \mathrm{~min}$ and then decreased linearly to $10 \%(\mathrm{v} / \mathrm{v})$ over $25 \mathrm{~min}$. Finally, mobile phase A was increased to $90 \%$ $(v / v)$ and the column was re-equilibrated for a further $9 \mathrm{~min}$.

The accuracy of natamycin extraction and quantification by RP-HPLC was determined by adding $2 \mathrm{~mL}$ agar samples with $100 \mu \mathrm{L}$ of a pure natamycin $(\geq 95 \%, w / w$, HPLC, SigmaAldrich, Munich, Germany) solution to obtain final concentrations in agar from 0.1 to $2.5 \mu \mathrm{g} \mathrm{mL}^{-1}$. Table 1 shows the natamycin rate recovery for each concentration tested.

Table 1 Accuracy of the natamycin extraction and quantification by RPHPLC

\begin{tabular}{lc}
\hline $\begin{array}{l}\text { Natamycin } \\
\text { concentration in agar } \\
\text { plates }\left(\mu \mathrm{g} \mathrm{mL}^{-1}\right)\end{array}$ & $\%$ Accuracy \\
\hline 2.50 & $78.80 \pm 2.83$ \\
1.00 & $79.00 \pm 1.41$ \\
0.50 & $96.00 \pm 11.31$ \\
0.25 & $120.00 \pm 11.31$ \\
0.10 & $207.00 \pm 18.38$ \\
\hline
\end{tabular}

The results of natamycin extraction and detection, shown in Table 1, are acceptable for natamycin concentrations above $0.25 \mu \mathrm{g} \mathrm{mL}^{-1}$. This detection limit (DL) was considered sufficient to address the natamycin release assays from the films with PNIPA/AA nanohydrogels, since minimum inhibitory concentrations (MIC) of natamycin are usually between 1 and $10 \mu \mathrm{g} \mathrm{mL}^{-1}$ or even higher for some moulds that, in general, are less sensitive than yeasts (Stark and Tan 2003; Delves-Broughton et al. 2010).

\section{Microbial Assay}

\section{Strains and Growth Conditions}

Stock cultures of Penicillium commune (P 1.01) were grown on potato dextrose agar (PDA, specific for cultivation of fungi, Difco $^{\mathrm{TM}}$, Detroit, MI, USA) acidified to $\mathrm{pH} 3.5$ with $10 \%(w /$ v) $\mathrm{L}(+)$-tartaric acid (Panreac, Barcelona, Spain) at $25^{\circ} \mathrm{C}$ and maintained on slants of this media at $4{ }^{\circ} \mathrm{C}$. The inoculum of this strain was prepared as explained in Fajardo et al. (2010) by overlaying mature slants with sterile Tween $80,0.05 \%(w /$ $v$ ), and gently scraping the surface with a Drigalski spatula. The mould culture suspensions were filtered through sterile gauze pads to remove the mycelium, and spore count was determined using a Neubauer chamber. Necessary dilutions were made with Tween $80,0.05 \%(w / v)$, to obtain $10^{6}$ spores per mL counts.

Stock cultures of Saccharomyces cerevisiae (Sc 1.02) were grown in YPD medium (yeast extract $10 \mathrm{~g} \mathrm{~L}^{-1}$; peptone $20 \mathrm{~g} \mathrm{~L}^{-1}$; $\mathrm{D}(+)$-glucose $20 \mathrm{~g} \mathrm{~L}^{-1}$, all from Panreac, Barcelona, Spain) at $25{ }^{\circ} \mathrm{C}$ and maintained at $-40{ }^{\circ} \mathrm{C}$ in the same media, supplemented with $15 \%(w / w)$ glycerol $(87 \%$, $v / v$, Panreac, Spain). The inoculum was prepared with a $12-\mathrm{h}$ culture after three steps, containing $26 \times 10^{6}$ colony-forming units (CFU) $\mathrm{mL}^{-1}$. Counts were determined by standard plate count with PDA acidified to $\mathrm{pH} 3.5$ with $10 \%(w / v) \mathrm{L}(+)$ tartaric acid.

\section{Inhibition Studies Employing a Food Model System}

Following the procedure described by Fuciños et al. (2012), a food model system consisting of Petri dishes with $5 \mathrm{~mL}$ of PDA acidified $(\mathrm{pH}=3)$ with $10 \%(w / v) \mathrm{L}(+)$-tartaric acid and plate storage under fluorescent lighting was used, in order to simulate unfavourable conditions for the chemical stability of natamycin. Three replicates of each film, prepared as explained in "Preparation of Natamycin-Loaded PNIPA/AA Nanohydrogels Incorporated into $\mathrm{K}$-Carrageenan and Locust Bean Gum (GA) Films", were evaluated.

$P$. commune (P 1.01) and S. cerevisiae (Sc 1.02) were used as indicator microorganisms. An aliquot of $25 \mu \mathrm{L}$ of a 12-h culture of Sc $1.02\left(26 \times 10^{6} \mathrm{CFU} \mathrm{mL}^{-1}\right)$ and $10 \mu \mathrm{L}$ of $\mathrm{P} 1.01$ $\left(26 \times 10^{6}\right.$ spores $\left.\mathrm{mL}^{-1}\right)$ was inoculated in triplicate into the 
Petri dishes. After $1 \mathrm{~h}$ in the fridge to allow the inoculated culture to be absorbed properly, films (GA, GA+NA(564), GA+NA(8), GA-PNIPA(5)+NA(8) and GA-PNIPA$20 \mathrm{AA}(5)+\mathrm{NA}(8))$ were placed covering the entire surface of the Petri dishes. All plates were incubated at $5{ }^{\circ} \mathrm{C}$ for 3 days and then at $37^{\circ} \mathrm{C}$, until the end of the experiment (8 days).

\section{Data Fitting and Statistical Analyses}

Student's $t$ test ( $\alpha=0.05$ ) was used to analyse differences between two samples for a single variable. One-way analysis of variance (ANOVA) followed by Tukey post-tests was performed for multiple comparisons. Both analyses were performed by using GraphPad Prism ${ }^{\mathrm{TM}} 5$ (GraphPad Software Inc., San Diego, CA, USA).

Plotting was also performed by using GraphPad Prism ${ }^{\mathrm{TM}}$ 5. Data fitting, parametric estimation and significance tests, both for parameters and models, were performed with SigmaPlot 11.0 (Systat Software, Inc. San Jose, CA, USA).

\section{Results and Discussion}

\section{Natamycin Release from PNIPA/AA Nanohydrogels Incorporated into GA Films}

Initially, natamycin diffusion from films to a liquid (distilled water) or solid medium (agar) was evaluated to determine the maximum release efficiency of the active compound from the system according to the application.

\section{Liquid Medium}

Taking into account that the natamycin release, during the dialysis process, will stop when the equilibrium concentration is reached, not all the natamycin loaded into the films could be recovered in the dialysis medium. That is why it has been considered that the maximum amount of natamycin released from the films is the amount of natamycin released in dialysis experiments carried out with free natamycin (Fuciños et al. 2014b). The relative fraction of natamycin released $\left(\gamma F_{t}\right)$ is defined by the following expression:

$\gamma F_{t}=\frac{C_{t} \text { GA-NA }}{C_{\max } \mathrm{NA}}$

where $C_{t}$ GA-NA is the amount of natamycin $(\mu \mathrm{g})$ released from the film to the dialysis medium (passing through the dialysis membrane) and $C_{\max } \mathrm{NA}$ is the amount of natamycin $(\mu \mathrm{g})$ under the steady-state conditions determined in the dialysis experiments with free natamycin (without film) for a given temperature. The amount of natamycin released through the dialysis membrane was not affected by temperature (data not shown) and was calculated with the equation developed in Fuciños et al. (2014b):

$C_{t}=C_{\max }+\left(C_{0}-C_{\max }\right) e^{-m\left(1+\sum_{i=1}^{k} \beta_{i} \cdot X_{i}\right) t}$

where $C_{t}$ is the concentration of the natamycin released $\left(\mu \mathrm{g} \mathrm{mL}^{-1}\right)$ at time $t(\mathrm{~min}), C_{0}$ is the initial natamycin concentration in the medium $\left(\mu \mathrm{g} \mathrm{mL}^{-1}\right), C_{\max }$ is the maximum achievable concentration of the released natamycin $\left(\mu \mathrm{g} \mathrm{mL}^{-1}\right), m$ is the maximum achievable release rate of natamycin $\left(\mathrm{min}^{-1}\right), k$ is the number of variables involved in a particular release system, $\beta_{i}$ is the coefficient which influences the $m$ and reflects the linear effect and $X_{i}$ represents the independent variable (temperature).

Figure 1 shows the natamycin release profiles obtained from GA films. The two most characteristic features of the natamycin release from GA films containing PNIPA/AA nanohydrogels (GA-PNIPA(5) and GA-PNIPA-20AA(5)) were that the release was controlled by the temperature and the kinetic shape was not hyperbolic, as expected from a simple diffusion process such as that observed for GA-NA cases (Fig. 1a, b). The lag time in Fig. 1c, d corresponds to the beginning of natamycin release. In addition, it seems that the temperature influences natamycin release from GA films, which increased when natamycin was incorporated into GA films by encapsulation into PNIPA/AA nanohydrogels (explained by the nanohydrogel collapse when the temperature rises above its lower critical solution temperature). This restricts natamycin release when the temperature is increased, i.e. when there is a risk of contamination/growth of microorganisms that cause food spoilage or the development of pathogenic microorganisms.

For a better analysis, experimental data were fitted with the reparametrised logistic model proposed by Vázquez et al. (2008) to make explicit the lag time:

$\gamma F_{t}=\frac{\gamma F_{\max }}{1+e^{2+\frac{4 \cdot v_{\max }}{\gamma F_{\max }}(\lambda-t)}}$

where $\gamma F_{\max }$ is the maximum relative fraction of natamycin released from the GA films through the dialysis membrane, $v_{\max }$ is the maximum release rate $\left(\mathrm{h}^{-1}\right)$ and $\lambda$ and $t$ are the lag time for the natamycin release and the sampling time, respectively, both expressed in hours (h).

Equation 3 provided a good fit of the experimental data and the parameters generated are shown in Table 2. Natamycin release from GA films when it was not nanoencapsulated (GANA(564)) appears to be governed only by molecular diffusion and by the polymer relaxation, as presented by Fajardo et al. (2010), and both phenomena will be influenced by temperature increase. The early time release data $(\sim 60 \%)$ of the natamycin release from the GA films studied were also fitted with the 
Fig. 1 Variation of the relative fraction of natamycin released $(\gamma F)$ with time from GA films loaded with free natamycin: GA+ $\mathrm{NA}(564)$ and $\mathrm{GA}+\mathrm{NA}(8)$ (a and b, respectively); and natamycin encapsulated into PNIPA/AA nanohydrogels: GA-PNIPA(5)+ NA(8) and GA-PNIPA$20 \mathrm{AA}(5)+\mathrm{NA}(8)(\mathbf{c}, \mathbf{d})$, through a 3500 -g-mol ${ }^{-1}$ cut-off dialysis membrane in distilled water at $10^{\circ} \mathrm{C}$ (empty circle) and $37^{\circ} \mathrm{C}$ (filled circle). The lines are the fitting curves generated from Eq. 3
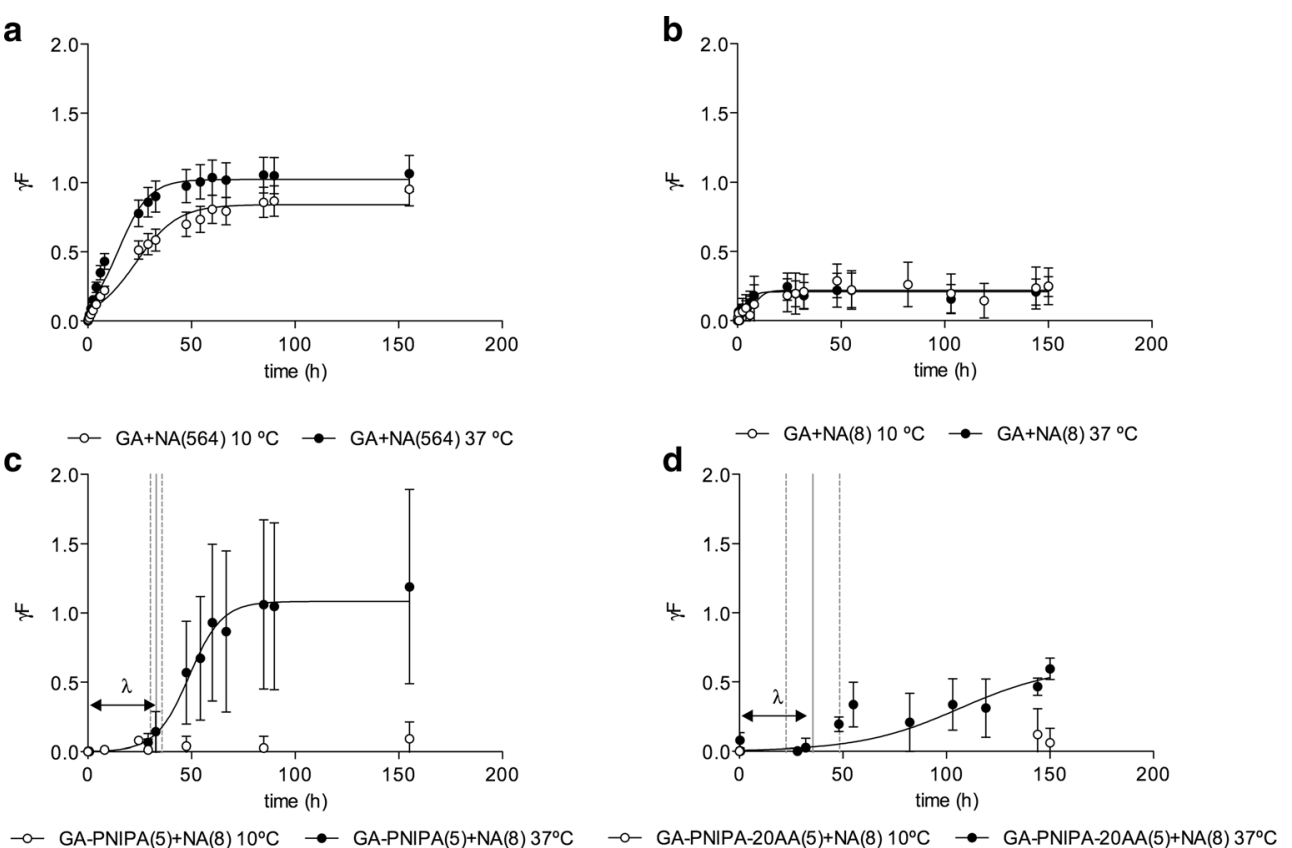

following well-known mathematical model (Ahuja et al. 2007; Blanco et al. 2008; Fuciños et al. 2014b):

$\gamma F_{t}=k \cdot t^{n}$

where $\gamma F_{t}$ is the relative fraction of natamycin released at time $t, k$ is a release kinetic constant that depends on the biomolecule-polymer system characteristics and $n$ is the diffusional exponent that depends on both the geometry of the delivery system and the physical mechanisms involved (Ritger and Peppas 1987). Thus, according to the $n$ value, the release from a thin film can be catalogued into one of the following diffusion mechanisms (Siepmann and Siepmann 2008):

- $n=0.5$ : Fickian diffusion (Case I). Biomolecule release is controlled only by diffusion into the release medium.
- $\quad n=1$ : Case II transport. Biomolecule release is controlled by the polymer swelling and relaxation mechanism.

- $\quad 0.5<n<1$ : Non-Fickian transport or anomalous transport. Biomolecule release is controlled by both mechanisms.

The values of diffusion exponent $n$ were $0.80 \pm 0.04$ and $0.66 \pm 0.04$ for natamycin release from GA film (GA$\mathrm{NA}(564))$ at 10 and $37{ }^{\circ} \mathrm{C}$, respectively, indicating that natamycin release from GA films was governed by polymer relaxation and natamycin diffusion into the release medium. The differences between these values are not significant $(p>0.05)$. However, due to the increase of the polymer relaxation rate with increasing temperatures (Bourbon et al. 2010), the natamycin release is favoured when temperature increases from 10 to $37^{\circ} \mathrm{C}$, showing significant $(p<0.05)$ differences in the values of $v_{\max }$ and $\gamma F_{\max }$ (Table 2).

Table 2 Natamycin release parameters from different GA films and temperatures, calculated with Eq. 3, in liquid medium

\begin{tabular}{|c|c|c|c|c|c|}
\hline Film & $\mathrm{T}\left({ }^{\circ} \mathrm{C}\right)$ & $\gamma F_{\max }$ & $v_{\max }\left(\mathrm{h}^{-1}\right)$ & $\lambda(\mathrm{h})$ & $r^{2}$ \\
\hline \multirow[t]{2}{*}{ GA-NA(564) } & 10 & $0.841 \pm 0.027 \mathrm{a}, \alpha$ & $0.021 \pm 0.002 \mathrm{a}, \alpha$ & $2.941 \pm 2.326$ n.s., a, $\alpha$ & 0.9716 \\
\hline & 37 & $1.024 \pm 0.028 \mathrm{~b}, \alpha$ & $0.035 \pm 0.004 \mathrm{~b}, \alpha$ & $-0.032 \pm 1.576$ n.s., a, $\alpha$ & 0.9712 \\
\hline \multirow[t]{2}{*}{$\mathrm{GA}+\mathrm{NA}(8)$} & 10 & $0.218 \pm 0.014 \mathrm{a}, \beta$ & $0.021 \pm 0.011$ n.s., $\mathrm{a}, \alpha$ & $2.735 \pm 2.048$ n.s., $\mathrm{a}, \alpha$ & 0.8348 \\
\hline & 37 & $0.209 \pm 0.011 \mathrm{a}, \beta$ & $0.023 \pm 0.006 \mathrm{a}, \alpha$ & $-0.145 \pm 1.093$ n.s., a, $\alpha$ & 0.8765 \\
\hline \multirow[t]{2}{*}{ GA-PNIPA(5)+NA(8) } & 10 & - & - & - & - \\
\hline & 37 & $1.084 \pm 0.040 \alpha$ & $0.034 \pm 0.005 \alpha$ & $32.914 \pm 2.797 \beta$ & 0.9798 \\
\hline \multirow[t]{2}{*}{ GA-PNIPA-20AA(5)+NA(8) } & 10 & - & - & - & - \\
\hline & 37 & $0.582 \pm 0.142 \gamma$ & $0.005 \pm 0.001 \beta$ & $35.438 \pm 12.942 \beta$ & 0.8364 \\
\hline
\end{tabular}

Values reported are the means \pm standard deviation $(n=2)$. a, b: statistically significant differences $(p<0.05)$ between different temperatures for the same film; $\alpha, \beta, \gamma$ : statistically significant differences $(p<0.05)$ between different films for the same temperature

n.s. parameter not significant $(p>0.05)$ 
Unlike GA-NA(564) films, when the concentration of natamycin was reduced to the same values as in the films with nanohydrogels $(\mathrm{GA}+\mathrm{NA}(8))$, it was not possible to extract all the natamycin loaded. This could be explained by a fixed amount of natamycin that always remains trapped in the films, for all the tested natamycin concentrations (Fig. 2). Contrary, if the initial amount of natamycin is very high, the fraction of natamycin remaining entrapped is imperceptible. Around $75 \%(w / w)$ of the natamycin initially charged remained trapped in the GA+ NA(8) film. However, this amount only accounts for around $1 \%(w / w)$ of the natamycin charged into the GA+NA(564) film.

The values of the diffusion exponent $n$ for $\mathrm{GA}+\mathrm{NA}(8)$ film were $0.75 \pm 0.34$ and $0.62 \pm 0.23$, at 10 and $37^{\circ} \mathrm{C}$, respectively. In the same manner as for the $\mathrm{GA}+\mathrm{NA}(564)$ films, there were no significant $(p>0.05)$ differences between the $n$ values at both temperatures assayed, showing that natamycin release from the films was governed by the polymer relaxation and natamycin diffusion into the release medium.

\section{$\mathrm{GA}+\mathrm{NA}(564)$}

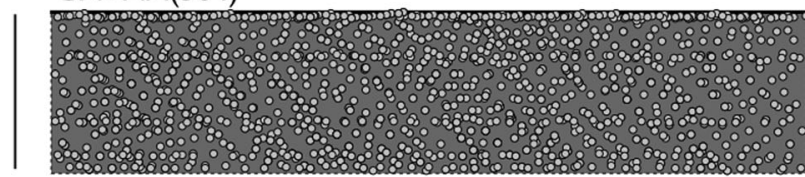

$\Delta T$

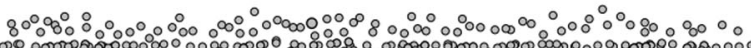

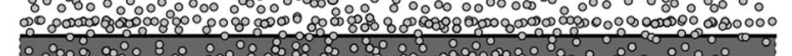

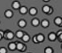

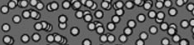
$\mathrm{GA}+\mathrm{NA}(8)$

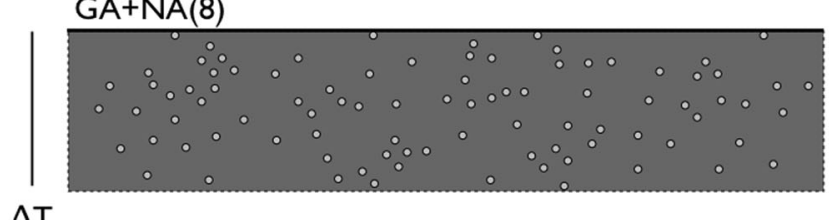

$\Delta T$

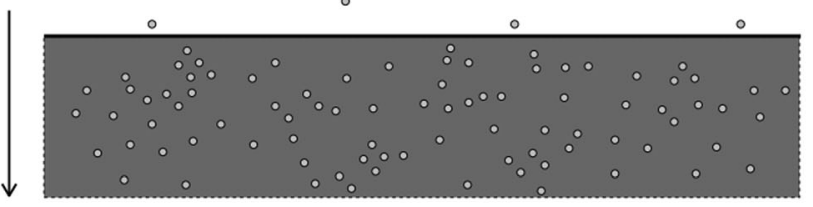

GA-PNIPA(5)+NA(8)/GA-PNIPA-20AA(5)+NA(8)

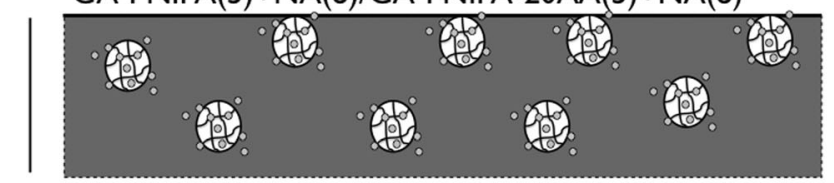

$\Delta T$

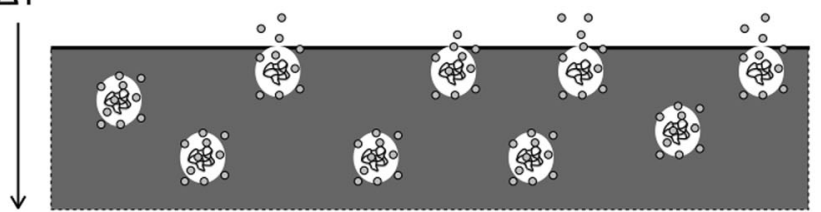

Fig. 2 Scheme of hypothetical behaviours to natamycin release, from different polymeric matrix, after a temperature rise
As mentioned above, the effect of temperature in natamycin release is more obvious when the natamycin is loaded into PNIPA/AA nanohydrogels and then incorporated into GA films. At $10{ }^{\circ} \mathrm{C}$, the release practically did not occur for both nanohydrogels assayed (Fig. 1), and for that reason, it was not possible to fit the experimental data (Table 2). The release was increased when the temperature was raised to $37^{\circ} \mathrm{C}$. These results are consistent with PNIPA behaviour, widely described in literature (Zhang et al. 2002; Huang and Lowe 2005; Fuciños et al. 2014a), where it is shown that when the temperature increases above the LCST, at ca. $33{ }^{\circ} \mathrm{C}$, hydrophobic interactions amongst PNIPA chains strengthen dramatically, leading to the collapse of the hydrogel and, consequently, to the release of the biomolecule.

The $\gamma F_{\max }$ value for the natamycin release at $37^{\circ} \mathrm{C}$ from GA-PNIPA(5)+NA(8) films was approximately 2 -fold $(p<0.05)$ the value for the natamycin release from GAPNIPA-20AA(5)+NA(8) films (Table 2). In addition, the $v_{\max }$ value was statistically different $(p<0.05)$ for both films with nanohydrogels. However, the $v_{\max }$ value for the GAPNIPA(5) +NA(8) films was not statistically different $(p<0.05)$ from films with free natamycin (i.e. GA-NA(564) and GA+NA(8)) (Table 2). This could be explained by the natamycin location near the surface or on the surface of nanohydrogel particles in the case of the nanohydrogel without AA (PNIPA(5)); when the collapse occurs at $37^{\circ} \mathrm{C}$, a fast release of natamycin occurs, leading to the burst effect (Fig. 1c). In contrast, natamycin could be more included into the most hydrophilic nanohydrogel matrix (PNIPA-20AA(5)). This behaviour is consistent with the results shown in Fuciños et al. (2012, 2014b), where it has been shown that when the nanoparticle collapses (with the increase of temperature), natamycin is released from inside the polymer matrix at a slower rate than when it remained near the polymer surface, justifying the slower and continuous release of natamycin from GA-PNIPA-20AA(5) films. The intense collapse, which occurred in GA-PNIPA(5) films when temperature increased, resulted in a rapid increase of natamycin release leading to a higher $\gamma F_{\max }$ value (Table 2) than that obtained with GAPNIPA-20AA(5) films, despite the release having stopped earlier (Fig. 1).

As explained before, there is a clear difference between the kinetic release of natamycin from GA films with and without nanohydrogels (Fig. 1). This fact was reflected in the statistical $(p<0.05)$ differences between their lag times (Table 2), increasing from values close to $0 \mathrm{~h}$ (i.e. the natamycin release began immediately) to values around $35 \mathrm{~h}$ when natamycin is added to the films loaded in the nanohydrogels. There were no significant differences between the lag time $(\lambda)$ with GA+ $\mathrm{NA}(564)$ and $\mathrm{GA}+\mathrm{NA}(8)$, and also there were no significant $(p>0.05)$ differences between the values of $\lambda$ with GAPNIPA(5)+NA(8) and GA-PNIPA-20AA(5)+NA(8). In Fuciños et al. (2014a), results showed that in a basic release 
medium ( $\mathrm{pH}>\mathrm{p} K_{\mathrm{a}}$ of $\left.\mathrm{AA}\right)$, it is difficult to find differences between the LCST value for the nanohydrogels with and without AA due to the presence of ionised $-\mathrm{COO}^{-}$groups, which prevent the collapse of nanohydrogels with AA. So, it is not strange that natamycin release due to the collapse of the nanohydrogel begins at the same time for both nanohydrogels, with and without AA, despite the more hydrophilic nanohydrogel collapse being less intense.

Finally, it is important to mention that the encapsulation of natamycin into nanohydrogels to be included into the GA films seems to help natamycin to be released when it was included in low quantities, since $\gamma F_{\max }$ with GA-PNIPA(5)+ $\mathrm{NA}(8)$ and GA-PNIPA-20AA(5)+NA(8) was significantly $(p<0.05)$ higher than $\gamma F_{\max }$ with $\mathrm{GA}+\mathrm{NA}(8)$, at $37^{\circ} \mathrm{C}$. This behaviour can be explained by the scheme presented in Fig. 2 , where the location of nanohydrogel particles near the film surface could favour natamycin release from films. Scanning electron microscopy (SEM) images of these films containing nanohydrogels, shown in a previous work (Cerqueira et al. 2014), seem to confirm the location of nanoparticles at the surface of films. So the encapsulation of natamycin into nanohydrogels (GA-PNIPA(5)+NA(8) and GA-PNIPA$20 \mathrm{AA}(5)+\mathrm{NA}(8))$ allows for a more concentrated presence of natamycin at certain points of the matrix (i.e. surface) compared to natamycin incorporated alone into the films $(\mathrm{GA}+\mathrm{NA}(8))$, facilitating natamycin diffusion from film matrix.

\section{Solid Medium}

In order to evaluate the amount of natamycin released from GA films with a different composition into a solid medium, i.e. agar, the fraction of natamycin released $\left(F_{t}\right)$ was calculated as the amount of natamycin extracted from the agar samples (as explained in "In Agar Plates"), in relation with the total amount of natamycin initially added to the film. Table 3 shows the $F_{t}$ values from different GA films and temperatures.

Lower fractions of natamycin were released to agar plates as the diffusion process in a solid medium is indeed far more complex than in a simple liquid medium. However, the release study in this matrix could provide a more realistic evaluation of the diffusion process in a food matrix.

In the same manner as in the liquid medium, the natamycin release from GA-NA(564) films increased and stabilised quickly for both temperatures (Table 3). However, in general, the $F_{t}$ values in the solid medium were statistically $(p<0.05)$ lower when temperature is increased to $37^{\circ} \mathrm{C}$, so it seems that natamycin release from these films was lower at higher temperatures, contrary to the results observed in a liquid medium. The behaviour was similar for the natamycin release from GA+ $\mathrm{NA}(8)$ films; however, the differences are not statistically significant $(p>0.05)$. In general, as in the liquid medium, lower $F_{t}$ values were obtained from this film compared with those $F_{t}$ values obtained with GA films initially loaded with high amounts of natamycin (Table 3).

Regarding natamycin release from PNIPA/AA nanohydrogels embedded into GA films, in general, $F_{t}$ values with GA-PNIPA $(5)+\mathrm{NA}(8)$ films were significantly $(p<0.05)$ higher than those obtained with GA-PNIPA-20AA(5)+NA(8) films, and as explained for the release kinetics in the liquid medium, this was probably due to a higher exposure of natamycin near the surface of nanohydrogel particles, as was concluded by Fuciños et al. (2012, 2014b). Nevertheless, in contrast to what happened in the liquid medium, it seems that the release only occurred at $5{ }^{\circ} \mathrm{C}$.

A control experiment was performed to check the availability of natamycin, both in distilled water (liquid medium) and agar plates (solid medium), during the storage time at temperatures used in natamycin release experiments. The results obtained (data not shown) indicate that the degradation or entrapment of natamycin in agar is not enough to explain the low natamycin release from films at $37^{\circ} \mathrm{C}$. There were no significant $(p>0.05)$ differences of natamycin concentration between samples stored at 10 and $37^{\circ} \mathrm{C}$ in water. And although significant $(p<0.05)$ differences appeared between samples stored at 10 and $37{ }^{\circ} \mathrm{C}$, differences only appeared over prolonged periods of time, in the experiment of natamycin availability in agar. However, low natamycin values were observed from $1 \mathrm{~h}$ of storage at $37^{\circ} \mathrm{C}$ (Table 3), in the experiments of natamycin release from films in agar. Therefore, the low water availability in the solid medium was proposed as the main cause of low natamycin release, slowing its diffusion from films. On the one hand, the absence of water reduces polymer relaxation, and therefore, the on/off release mechanism of PNIPA nanohydrogels could be limited; on the other hand, the absence of water at high temperatures could favour the formation of hydrophobic interactions between natamycin and polymer chains, as noted in Fuciños et al. (2014b) to explain a great natamycin entrapment in PNIPA nanohydrogels when the nanoparticles expelled the water contained in them.

\section{Antimicrobial Efficiency of Natamycin-Loaded PNIP A/AA Nanohydrogels Incorporated into GA Films}

In Fuciños et al. (2012), the protection capacity of PNIPA/ AA nanohydrogels has been demonstrated, which allowed natamycin to remain active under conditions that were unfavourable for its chemical stability, thus improving its antimicrobial effect. That is why, in this work, we also evaluated the antimicrobial effectiveness of GA films containing natamycin-loaded nanohydrogels under the same unfavourable conditions for natamycin stability employed in Fuciños et al. (2012), i.e. acidified PDA plates and fluorescent lighting.

In plates inoculated with the indicator mould ( $\mathrm{P}$ 1.01 ), growth appears to be limited by the coating itself, 
Table 3 Fraction of natamycin released $\left(F_{t}\right)$ from different GA films and temperatures in solid medium

\begin{tabular}{|c|c|c|c|c|c|}
\hline \multirow[t]{2}{*}{ Storage time $(\mathrm{h})$} & \multirow[t]{2}{*}{$\mathrm{T}\left({ }^{\circ} \mathrm{C}\right)$} & \multicolumn{4}{|l|}{ Film } \\
\hline & & $\mathrm{GA}+\mathrm{NA}(564)$ & $\mathrm{GA}+\mathrm{NA}(8)$ & GA-PNIPA(5)+NA(8) & GA-PNIPA-20AA(5)+NA(8) \\
\hline \multirow[t]{2}{*}{1} & 5 & $0.029 \pm 0.006 \mathrm{a}, \alpha$ & $0.025 \pm 0.016 \mathrm{a}, \alpha$ & $0.053 \pm 0.015 \mathrm{a}, \beta$ & $<\mathrm{DL}$ \\
\hline & 37 & $0.049 \pm 0.011 \mathrm{~b}, \alpha$ & $0.009 \pm 0.013 \mathrm{a}, \beta$ & $<\mathrm{DL}$ & $<\mathrm{DL}$ \\
\hline \multirow[t]{2}{*}{2} & 5 & $0.068 \pm 0.015 \mathrm{a}, \alpha$ & $0.025 \pm 0.029 a, \alpha, \beta$ & $0.071 \pm 0.033 \mathrm{a}, \alpha$ & $<\mathrm{DL}$ \\
\hline & 37 & $0.038 \pm 0.008 b, \alpha$ & $0.020 \pm 0.023 \mathrm{a}, \alpha, \beta$ & $<\mathrm{DL}$ & $<\mathrm{DL}$ \\
\hline \multirow[t]{2}{*}{4} & 5 & $0.045 \pm 0.009 \mathrm{a}, \alpha$ & $0.029 \pm 0.035 \mathrm{a}, \alpha, \beta$ & $0.058 \pm 0.012 \mathrm{a}, \alpha$ & $<\mathrm{DL}$ \\
\hline & 37 & $0.049 \pm 0.012 \mathrm{a}, \alpha$ & $0.006 \pm 0.011 \mathrm{a}, \beta$ & $<\mathrm{DL}$ & $<\mathrm{DL}$ \\
\hline \multirow[t]{2}{*}{8} & 5 & $0.057 \pm 0.007 \mathrm{a}, \alpha$ & $<\mathrm{DL}$ & $0.036 \pm 0.012 \mathrm{a}, \gamma$ & $<\mathrm{DL}$ \\
\hline & 37 & $0.033 \pm 0.003 b, \alpha$ & $0.008 \pm 0.015 \mathrm{a}, \beta$ & $<\mathrm{DL}$ & $<\mathrm{DL}$ \\
\hline \multirow[t]{2}{*}{22} & 5 & $0.069 \pm 0.013 \mathrm{a}, \alpha$ & $0.027 \pm 0.053 \mathrm{a}, \alpha, \beta$ & $0.040 \pm 0.014 \mathrm{a}, \alpha, \beta$ & $<\mathrm{DL}$ \\
\hline & 37 & $0.034 \pm 0.009 \mathrm{~b}, \alpha$ & $0.008 \pm 0.016 \mathrm{a}, \alpha$ & $<\mathrm{DL}$ & $0.015 \pm 0.029 \mathrm{a}, \alpha$ \\
\hline \multirow[t]{2}{*}{30} & 5 & $0.068 \pm 0.007 \mathrm{a}, \alpha$ & $0.102 \pm 0.013 \mathrm{a}, \beta$ & $0.058 \pm 0.018 \mathrm{a}, \alpha$ & $<\mathrm{DL}$ \\
\hline & 37 & $0.044 \pm 0.010 \mathrm{~b}, \alpha$ & $0.042 \pm 0.031 b, \alpha$ & $<\mathrm{DL}$ & $<\mathrm{DL}$ \\
\hline \multirow[t]{2}{*}{46} & 5 & $0.060 \pm 0.013 \mathrm{a}, \alpha$ & $0.014 \pm 0.028 \mathrm{a}, \beta$ & $0.060 \pm 0.012 \mathrm{a}, \alpha$ & $<\mathrm{DL}$ \\
\hline & 37 & $0.023 \pm 0.008 \mathrm{~b}, \alpha$ & $<\mathrm{DL}$ & $<\mathrm{DL}$ & $<\mathrm{DL}$ \\
\hline \multirow[t]{2}{*}{70} & 5 & $0.046 \pm 0.012 \mathrm{a}, \alpha$ & $0.015 \pm 0.018 \mathrm{a}, \beta$ & $<\mathrm{DL}$ & $<\mathrm{DL}$ \\
\hline & 37 & $0.031 \pm 0.016 \mathrm{a}, \alpha$ & $0.008 \pm 0.016 \mathrm{a}, \alpha, \beta$ & $<\mathrm{DL}$ & $<\mathrm{DL}$ \\
\hline
\end{tabular}

Values reported are the means \pm standard deviation $(n=4)$. a, b: statistically significant differences $(p<0.05)$ between different temperatures for the same film; $\alpha, \beta, \gamma$ : statistically significant differences $(p<0.05)$ between different films for the same temperature

since there is virtually no growth throughout the storage time and there were no noticeable differences between films with the antimicrobial treatments and controls (Fig. 3).
The plates inoculated with the indicator yeast ( $\mathrm{Sc} 1.02)$ provide more revealing results that are consistent with the results observed in the release experiments into a solid medium (see "Solid Medium"). So, no growth was observed at

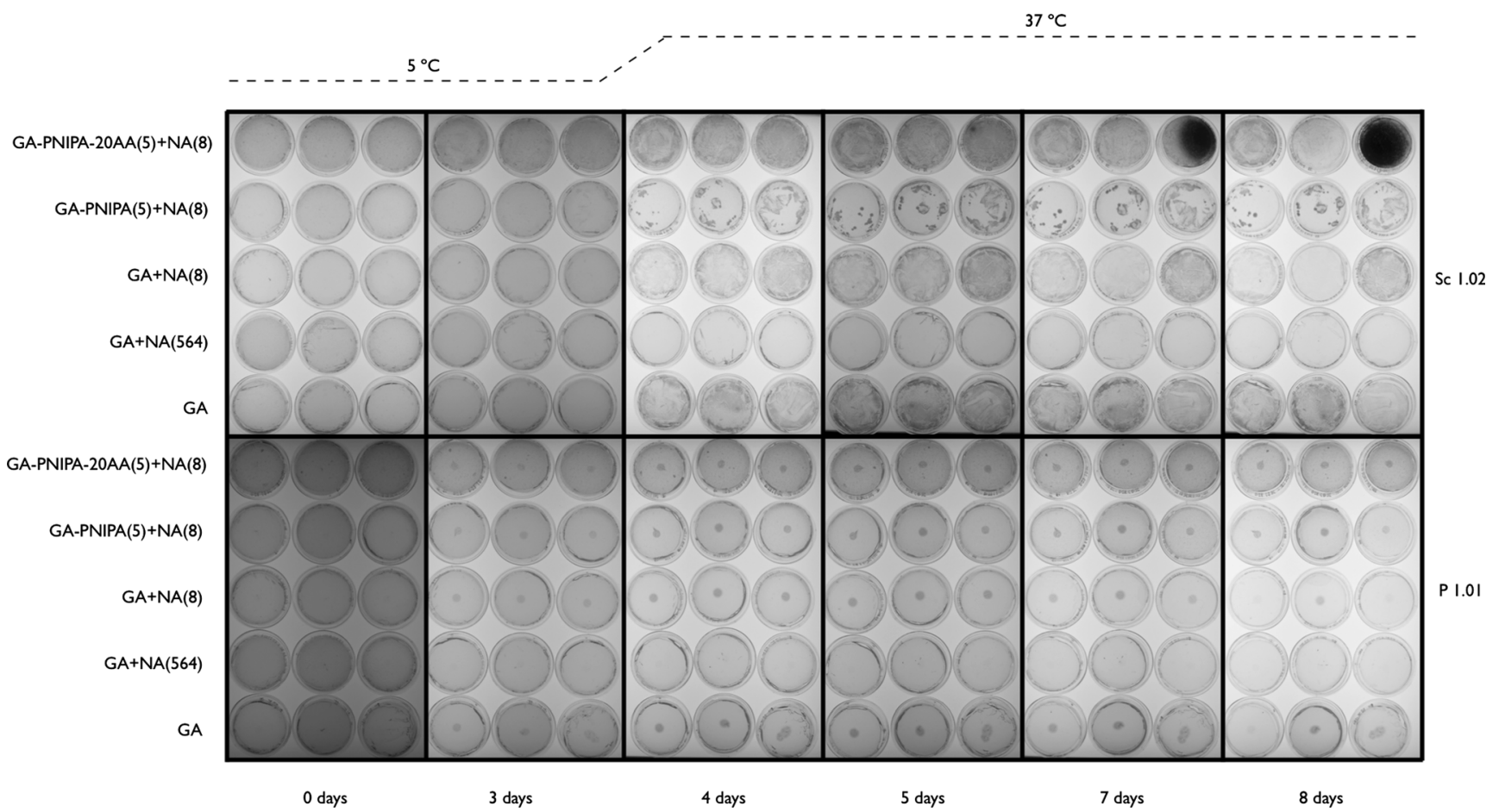

Fig. 3 Inhibition of Saccharomyces cerevisiae (Sc 1.02) and Penicillium commune (P 1.01) in acidified PDA agar plates by treatment with different GA films, under fluorescent lighting in order to simulate unfavourable conditions for the chemical stability of natamycin 
$5{ }^{\circ} \mathrm{C}$ and growth peaked rapidly when the temperature increased up to $37{ }^{\circ} \mathrm{C}$ in control plates treated with GA films without natamycin (GA) (Fig. 3). In plates treated with films that have the highest natamycin content (GA-NA(564)), inhibition was maintained until the end of the assay ( 8 days). By contrast, higher growth was observed in those plates treated with GA-PNIPA-20AA(5)+NA(8) films, according to the lower natamycin release observed from these films to agar plates (Table 3). Growth observed with the GA-PNIPA(5)+ $\mathrm{NA}(8)$ and GA+NA(8) films was less intense, when compared with GA-PNIPA-20AA(5)+NA(8) films, especially for the first one (Fig. 3). Results showed plates with large inhibition zones when these were treated with GAPNIPA(5)+NA(8) films when compared with those plates treated with GA and GA+NA(8) films. These differences could be due to the protection of natamycin against degradation conditions, i.e. under acidic conditions (Stark and Tan 2003) and fluorescent lighting (Koontz et al. 2003), when it was included into the nanohydrogels allowing its release only when the temperature increased (Fuciños et al. 2012). This makes it possible to maintain the inhibition for more days.

\section{Conclusions}

In view of the results observed in both systems - liquid and solid medium - it is necessary to continue working to improve the amount of natamycin released from the new smart films where it seems that the PNIPA/AA nanohydrogel swelling/ collapse is limited once it was trapped into the GA films. In any case, this system makes it possible to reduce natamycin concentrations in food products whilst improving the antifungal effect in terms of that observed when natamycin was included alone in the GA film, by the same amount. We noted promising results that demonstrate the potential of polysaccharide-based films containing smart nanohydrogels for the controlled release of food preservatives.

\footnotetext{
Acknowledgments Clara Fuciños and Miguel A. Cerqueira are recipients of a fellowship (SFRH/BPD/87910/2012 and SFRH/BPD/72753/ 2010, respectively) from the Fundação para a Ciência e Tecnologia (FCT, POPH-QREN, and FSE Portugal). The authors thank the FCT Strategic Project PEst-OE/EQB/LA0023/2013 and the project "BioInd - Biotechnology and Bioengineering for improved Industrial and Agro-Food processes", Ref. NORTE-07-0124-FEDER-000028 co-funded by the Programa Operacional Regional do Norte (ON.2 - O Novo Norte), QREN, FEDER and the project from the "Ministerio de Educación y Ciencia" (Spain) "Nanohidrogeles inteligentes sensibles a cambios de pH y Temperatura: Diseño, síntesis y aplicación en terapia del cáncer y el envasado activo de alimentos", Ref. MAT2010-21509-C03-01.
}

\section{References}

Ahuja, N., Katare, O. P., \& Singh, B. (2007). Studies on dissolution enhancement and mathematical modeling of drug release of a poorly water-soluble drug using water-soluble carriers. European Journal of Pharmaceutics and Biopharmaceutics, 65(1), 26-38.

Bierhalz, A. C. K., Da Silva, M. A., \& Kieckbusch, T. G. (2012). Natamycin release from alginate/pectin films for food packaging applications. Journal of Food Engineering, 110(1), 18-25.

Blanco, M. D., Guerrero, S., Teijón, C., Olmo, R., Pastrana, L., Katime, I., et al. (2008). Preparation and characterization of nanoparticulate poly $(N$-isopropylacryl-amide) hydrogel for the controlled release of anti-tumour drugs. Polymer International, 57(11), 1215-1225.

Bourbon, A. I., Pinheiro, A. C., Ribeiro, C., Miranda, C., Maia, J. M., Teixeira, J. A., et al. (2010). Characterization of galactomannans extracted from seeds of Gleditsia triacanthos and Sophora japonica through shear and extensional rheology: comparison with guar gum and locust bean gum. Food Hydrocolloids, 24(2-3), 184-192.

Brody, A. L., Bugusu, B., Han, J. H., Sand, C. K., \& McHugh, T. H. (2008). Innovative food packaging solutions. Journal of Food Science, 73(8), R107-R116.

Cerqueira, M. A., Costa, M. J., Fuciños, C., Pastrana, L. M., \& Vicente, A. A. (2014). Development of active and nanotechnology-based smart edible packaging systems: physical-chemical characterization. Food and Bioprocess Technology, 7(5), 1472-1482.

Cordeiro, A. L., Zimmermann, R., Gramm, S., Nitschke, M., Janke, A., Schäfer, N., et al. (2009). Temperature dependent physicochemical properties of poly $(N$-isopropylacrylamide-co- $N$-(1-phenylethyl) acrylamide) thin films. Soft Matter, 5(7), 1367-1377.

Dainelli, D., Gontard, N., Spyropoulos, D., Zondervan-van den Beuken, E., \& Tobback, P. (2008). Active and intelligent food packaging: legal aspects and safety concerns. Trends in Food Science and Technology, 19(SUPPL. 1), S99-S108.

Delves-Broughton, J., Steenson, L., Dorko, C., Erdmann, J., Mallory, S., Norbury, F., et al. (2010). Use of natamycin as a preservative on the surface of baked goods: a case study. In C. J. Doona, K. Kustin, \& F. E. Feeherry (Eds.), Case studies in novel food processing technologies: innovations in processing, packaging, and predictive modelling (pp. 303-320). UK: Woodhead Publishing.

Fajardo, P., Martins, J. T., Fuciños, C., Pastrana, L., Teixeira, J. A., \& Vicente, A. A. (2010). Evaluation of a chitosan-based edible film as carrier of natamycin to improve the storability of Saloio cheese. Journal of Food Engineering, 101(4), 349-356.

Farid, M. A., El-Enshasy, H. A., El-Diwany, A. I., \& El-Sayed, E. A. (2000). Optimization of the cultivation medium for natamycin production by Streptomyces natalensis. Journal of Basic Microbiology, 40(3), 157-166.

Fuciños, C., Guerra, N. P., Teijón, J. M., Pastrana, L. M., Rúa, M. L., \& Katime, I. (2012). Use of poly( $N$-isopropylacrylamide) nanohydrogels for the controlled release of pimaricin in active packaging. Journal of Food Science, 77(7), N21-N28.

Fuciños, C., Fuciños, P., Míguez, M., Katime, I., Pastrana, L. M., \& Rúa, M. L. (2014a). Temperature- and $\mathrm{pH}$-sensitive nanohydrogels of poly( $N$-Isopropylacrylamide) for food packaging applications: modelling the swelling-collapse behaviour. PLoS ONE, 9(2), e87190.

Fuciños, C., Fuciños, P., Pastrana, L. M., \& Rúa, M. L. (2014b). Functional characterization of poly $(N$-isopropylacrylamide $)$ nanohydrogels for the controlled release of food preservatives. Food and Bioprocess Technology, 7(12), 3429-3441.

Han, J. H. (2005). New technologies in food packaging: overview. In J. H. Han (Ed.), Innovations in food packaging (pp. 3-11). UK: Elsevier Academic Press. 
Han, J. H., Ho, C. H. L., \& Rodrigues, E. T. (2005). Intelligent packaging. In J. Han (Ed.), Innovations in food packaging (pp. 138-155). UK: Elsevier Academic Press.

Hanušová, K., Št’astná, M., Votavová, L., Klaudisová, K., Dobiáš, J., Voldřich, M., et al. (2010). Polymer films releasing nisin and/or natamycin from polyvinyldichloride lacquer coating: nisin and natamycin migration, efficiency in cheese packaging. Journal of Food Engineering, 99(4), 491-496.

Huang, X., \& Lowe, T. L. (2005). Biodegradable thermoresponsive hydrogels for aqueous encapsulation and controlled release of hydrophilic model drugs. Biomacromolecules, 6(4), 2131-2139.

Koontz, J. L., Marcy, J. E., Barbeau, W. E., \& Duncan, S. E. (2003). Stability of natamycin and its cyclodextrin inclusion complexes in aqueous solution. Journal of Agricultural and Food Chemistry, 51(24), 7111-7114.

Ritger, P. L., \& Peppas, N. A. (1987). A simple equation for description of solute release II. Fickian and anomalous release from swellable devices. Journal of Controlled Release, 5(1), 37-42.

Roberts, D. P. T., Scotter, M. J., Godula, M., Dickinson, M., \& Charlton, A. J. (2011). Development and validation of a rapid method for the determination of natamycin in wine by high-performance liquid chromatography coupled to high resolution mass spectrometry. Analytical Methods, 3(4), 937-943.

Rooney, M. L. (2005). Introduction to active food packaging technologies. In J. Han (Ed.), Innovations in food packaging (pp. 63-79). UK: Elsevier Academic Press.

Schild, H. G. (1992). Poly( $N$-isopropylacrylamide): experiment, theory and application. Progress in Polymer Science (Oxford), 17(2), 163-249.
Schmaljohann, D. (2006). Thermo- and pH-responsive polymers in drug delivery. Advanced Drug Delivery Reviews, 58, 1655-1670.

Sekhon, B. S. (2010). Food nanotechnology-an overview. Nanotechnology, Science and Applications, 3, 1-15.

Siepmann, J., \& Siepmann, F. (2008). Mathematical modeling of drug delivery. International Journal of Pharmaceutics, 364(2), 328-343.

Stark, J., \& Tan, H. S. (2003). Natamycin. In N. J. Russell \& G. W. Gould (Eds.), Food preservatives (pp. 179-195). USA: Kluwer Academic/ Plenum Publishers.

Türe, H., Eroğlu, E., Özen, B., \& Soyer, F. (2009). Physical properties of biopolymers containing natamycin and rosemary extract. International Journal of Food Science and Technology, 44(2), 402-408.

Vanden Bossche, H., Engelen, M., \& Rochette, F. (2003). Antifungal agents of use in animal health - chemical, biochemical and pharmacological aspects. Journal of Veterinary Pharmacology and Therapeutics, 26(1), 5-29.

Vázquez, J. A., Docasal, S. F., Prieto, M. A., González, M. P., \& Murado, M. A. (2008). Growth and metabolic features of lactic acid bacteria in media with hydrolysed fish viscera. An approach to biosilage of fishing by-products. Bioresource Technology, 99(14), 6246-6257.

Vermeiren, L., Devlieghere, F., Van Beest, M., De Kruijf, N., \& Debevere, J. (1999). Developments in the active packaging of foods. Trends in Food Science and Technology, 10(3), 77-86.

Zhang, X., Zhuo, R., Cui, J., \& Zhang, J. (2002). A novel thermoresponsive drug delivery system with positive controlled release. International Journal of Pharmaceutics, 235(1-2), 43-50. 